\title{
Design and Experimental Research of a New Type of Asteroid Anchoring System
}

\author{
Wang Yongbin, ${ }^{1,2,3}$ Jiang Wansong $\mathbb{B}^{2,3}$ Long Long, ${ }^{2,3}$ Zhu Qian, ${ }^{2,3}$ Feng Rui, ${ }^{2,3}$ \\ and Wang Liwu ${ }^{2,3,4}$ \\ ${ }^{1}$ College of Astronautics, Nanjing University of Aeronautics and Astronautics, Nanjing 210016, China \\ ${ }^{2}$ Beijing Institute of Space Mechanics \& Electricity, Beijing 100094, China \\ ${ }^{3}$ Laboratory of Aerospace Entry, Descent and Landing Technology, CASC, Beijing 100094, China \\ ${ }^{4}$ Southeast University, School of Civil Engineering, Nanjing 210096, China
}

Correspondence should be addressed to Jiang Wansong; gaokaozs2@163.com

Received 20 November 2020; Revised 24 March 2021; Accepted 12 April 2021; Published 19 May 2021

Academic Editor: Antonio Concilio

Copyright ( 2021 Wang Yongbin et al. This is an open access article distributed under the Creative Commons Attribution License, which permits unrestricted use, distribution, and reproduction in any medium, provided the original work is properly cited.

Asteroid detection is of great significance to the study of the formation of the solar system and the origin of life. However, there are many types of asteroids, and they are far away from the earth, and the understanding of their various characteristics is not clear, which brings huge technical challenges to the landing and attachment of star catalogs. At present, the world is mainly based on surround, overflight, and short-term contact detection, and long-term attachment detection has not yet been realized. In order to solve the long-term attachment detection requirements of asteroids, focusing on the geological characteristics of various types of stars, this paper proposes a new type of asteroid attachment mechanism based on the beetle bionic theory, which can realize intelligent and flexible attachment and has strong adaptability. Around this design, this paper analyzes the mechanism of adhesion and realizes the adaptive matching of unascertained terrain landing point adhesion. On this basis, a prototype of the asteroid landing attachment mechanism was developed and verified by experiments. The experiment proved that the mechanism has strong multiterrain matching ability and can obtain an adhesion force of not less than $36 \mathrm{~N}$ on ordinary concrete ground.

\section{Introduction}

The detection of small celestial bodies is an important part of the field of deep space exploration and one of the most active technical fields in the development of space technology. The results can provide clues and basis for the formation and evolution of the solar system and have important guiding significance for studying the life information of the solar system and exoplanets, studying the evolution of terrestrial planets and the solar system, and revealing the origin of Earth's biology; its rich scarce substances. Resources may become a treasure house for human development and utilization in the future $[1,2]$. The "General Implementation Plan for my country's Deep Space Exploration Before 2030" issued by the National Defense Science and Industry Bureau clearly includes the small celestial body detection mission, and it is proposed that Mars sampling return, asteroid exploration, and Jupiter system exploration should be realized around 2030.

At present, space powers such as the United States, Europe, and Japan have achieved a series of results in asteroid detection [3-7]. From the perspective of the development of deep space exploration technology abroad, the detection of planets and small celestial bodies mainly adopts methods such as short-range leap, surround detection, and surface landing attachment survey [8-17]. Among them, the attachment detection method is the most direct, and the detection efficiency is high, but the technical difficulty and risk are also high. The detection of small celestial bodies is relatively large, and the detection of small celestial bodies is still facing the problem of the unstructured extremely harsh space environment. Therefore, it is very necessary to carry out research on 
asteroid landing attachment. Based on the adhesion technology, the small object detector can be reliably fixed on the surface of the weak gravitational object to prevent the small object detector from flying off the surface of the object caused by the external disturbance, which can provide the working conditions such as adhesion and torque for the drilling, sampling, and other scientific equipment. At present, scholars have proposed various attachment techniques such as anchoring, grasping, dry adhesion, or drilling, but how to construct an attachment system with high reliability, high adaptability, and intelligent attachment is urgently needed to carry out related research.

\section{Current Research Status and Trend Analysis at Home and Abroad}

Focusing on the detection of small celestial bodies, the United States, ESA, the former Soviet Union, and Japan have carried out 18 types of detections since 1978, including crossing, orbiting, and landing detections [3-7]. Among them, the only spacecraft that can actually explore the surface of small celestial bodies is Japan's Hayabusa probe and ESA's Rosetta probe, while the only one that achieves true landing attachment detection is the "Rosetta" detector.

As shown in Figure 1, the Hayabusa 1 probe was launched in May 2003, and landed on the asteroid "Ishikawa" in November 2005, and collected splashed rock and soil samples in June 2010. Successfully returned to Earth, the mission obtained almost 1,500 samples of asteroid particles. The Hayabusa 2 probe was launched in December 2014 and dropped to the surface of the Dragon Palace in February 2019. It is expected to return to Earth by the end of 2020. Hayabusa uses a $300 \mathrm{~m} / \mathrm{s}$ projectile before landing and collects samples from the impact of the projectile through a sample collector. This type of detection method can be attributed to contact detection instead of landing attachment detection [8-11].

Figure 2 shows the "Rosetta" probe, which is the world's first probe aimed at landing on the surface of a small celestial body. It was launched in March 2004 and entered 67P/Churiu in August 2014. Move-Gerasimenko orbited the asteroid and subsequently released the Philae lander in November [12]. The lander uses electromagnetic damping buffers to absorb landing impact energy. As shown in Figures 3 and 4, the iced bolts at the bottom of the foot pads and the harpoon anchoring device are used to anchor the asteroids. The above three technologies are used for matching and coupling. That is, the electromagnetic damping buffer is used to self-adjust the attitude and absorb energy, and then the iced bolt is used to contact the landing surface and anchor, and finally, the harpoon is used to anchor. The iced bolt uses the initial energy of the landing impact to generate bolt rotation through the rope system to achieve drilling. The harpoon uses the power of gunpowder combustion to achieve high-speed impact penetration into the asteroid and uses the barb to achieve anchoring. Unfortunately, in September 2016, the "Philae" lander failed to land and lost contact with the ground [13-17].

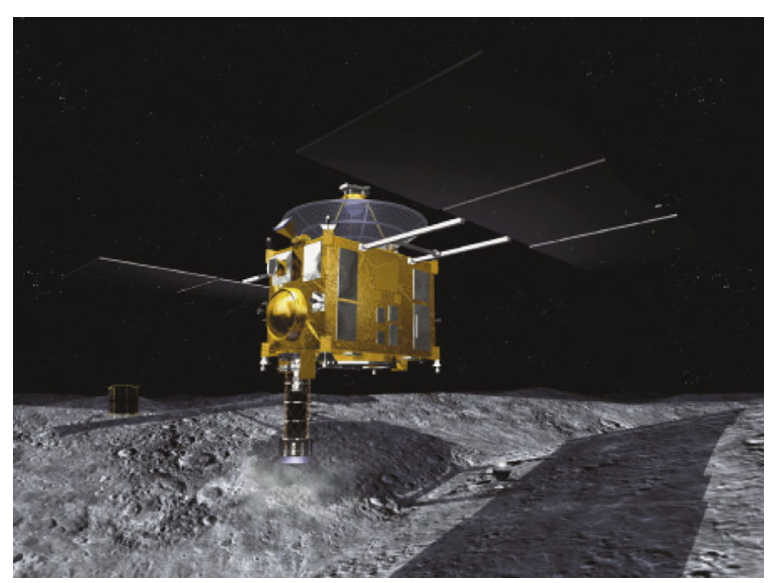

Figure 1: Japanese Hayabusa explorer.

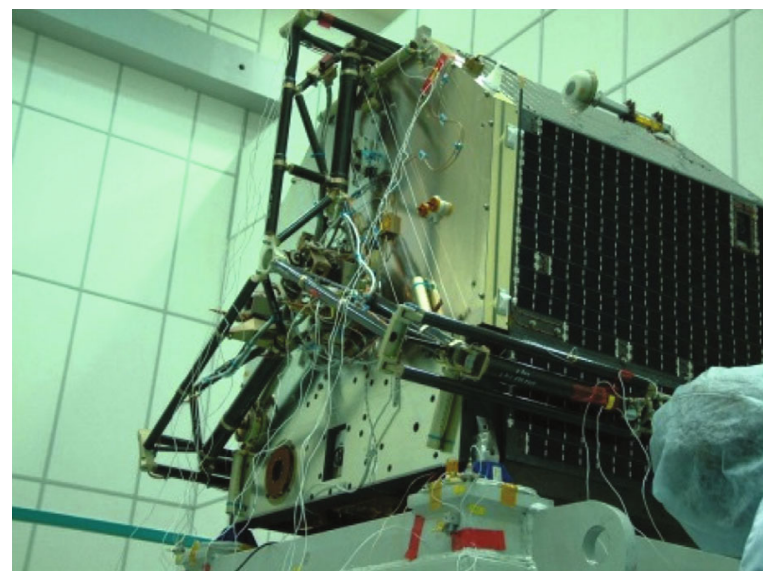

Figure 2: Rosetta probe.

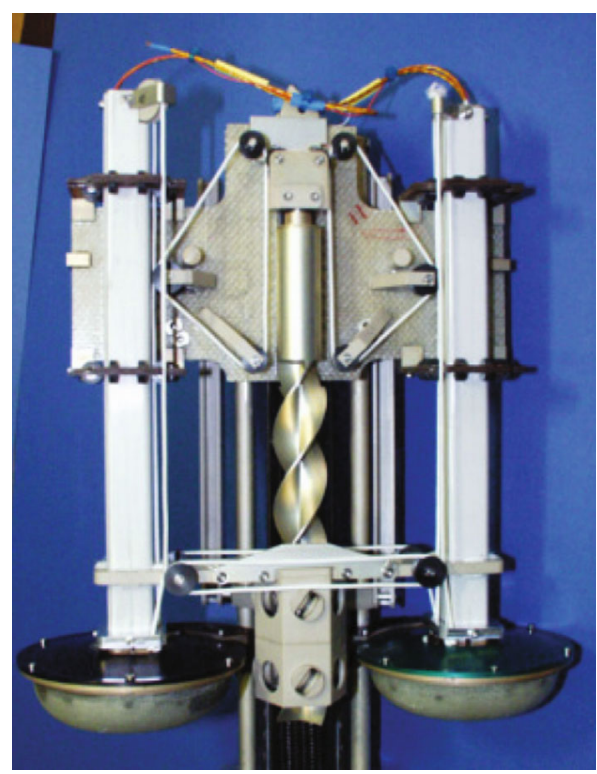

FIGURE 3: Icy bolt anchoring device.

In addition to the above two attachment systems verified by onorbit flights, many research institutions have carried out systematic and indepth research on attachment detection 


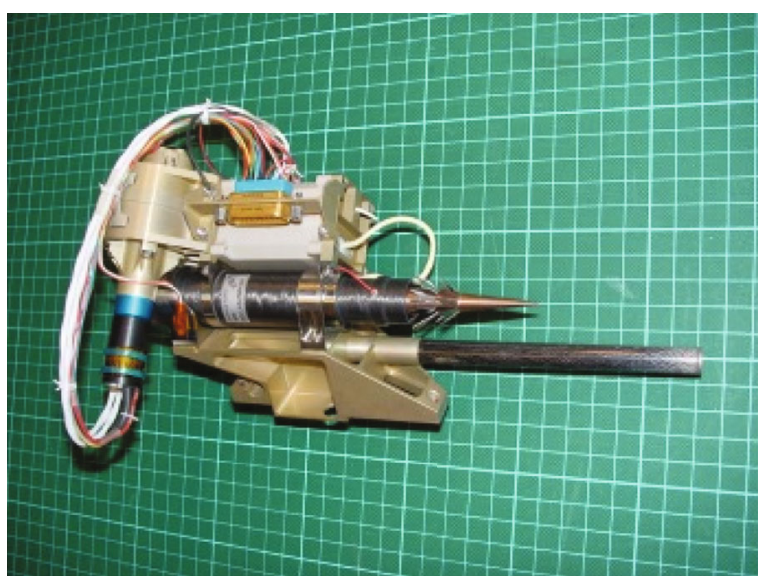

FIgURE 4: Harpoon anchoring device.

methods for unknown star catalogs. As shown in Figure 5, a small tip attachment system based on elastic elements was proposed by NASA. The original intention of the system was to be applied to wall-climbing robots. NASA's Jet Propulsion Laboratory extended it to the catalog of small celestial bodies. The design uses elastic elements as the flexible suspension system and uses steel hooks to hook the star and watch, and omnidirectional attachment can be achieved through the array of 16 anchor components. Experiments show that it can provide an adhesion of $120 \mathrm{~N}$ and can be designed according to the needs of several to thousands of new Newtonian adhesion forces. The robot based on the claw-stick attachment technology can be used to transform the end effector of the spacecraft manipulator, or the handle used by astronauts to land on asteroids or the Moon. It can work in a microgravity environment and is also suitable for the steep surface of Mars or the Moon Tour [16, 18]; other anchoring systems include the anchoring system based on uniform back drilling developed by the Bee Robot Company, which will be applied to the NASA exploration mission of Amor; in addition, the magnetic-based anchoring system research of technical methods such as anchoring, net enveloping, hard rock beating, bonding, and gecko bionic adhesion, has promoted the diversity of anchoring schemes [16, 17].

Compared with a number of existing adhesion technologies, the dry adhesion technology of imitation gecko has been studied in recent years, which can achieve adsorption on smooth walls, but the adhesive materials are easily contaminated; electrostatic adsorption requires high input voltage and low suction force; adsorption requires that the wall surface must have body magnetism, which is often used for man-made targets; suction cup adsorption is the most common, but it is prone to gas leakage and failure on the wall with cracks or uneven walls, which is not suitable for space environment; the imitation beetle attachment technology can be used on rough hard surfaces. Attachment has attracted the attention of domestic and foreign researchers in recent years. This technology can adapt to the surface environment of hard small celestial bodies.

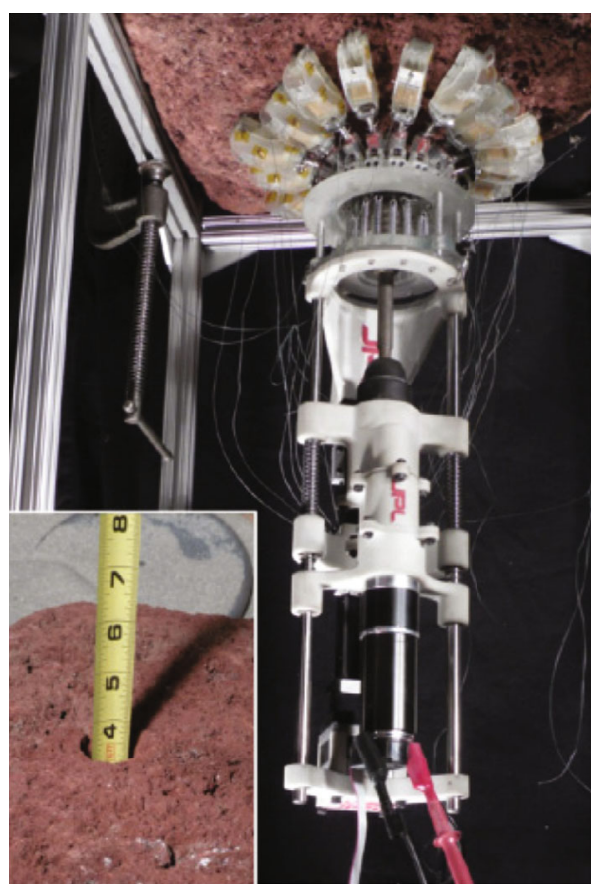

Figure 5: Microspine anchor device by elastic element.

\section{Design of New Attachment Mechanism for Small Celestial Bodies}

One of the main functions of the attachment system for small celestial bodies is to provide a set force or moment against the contact impact force through the attachment system, which is convenient for subsequent detectors to carry out in situ detection. Since the small celestial body to be landed is too far from the Earth to predict the physical characteristics of the landing site in advance, the attachment system needs to overcome and adapt to a variety of environments, including fluffy powdery star tables, broken gravel, and hard rocks.

3.1. Design and Composition of the Claw Attachment System. Based on the above attachment requirements, a bionic beetlebased claw thorn attachment system is proposed. The system uses a nonphysical damage to the surface structure to achieve the attachment of the hard asteroid surface structure, prevent the asteroid lander from rebounding and drifting away after landing, and provide the required adhesion or moment for scientific detection loads such as drilling and sampling. As shown in Figure 6, the claw attachment system consists of a universal connection mechanism, a grasping reset motor and 16 sets of claw grasping mechanism arrays.

The claw thorn array is a circular array of 6 thorns/ group $\times 16$ groups, a total of 8 pairs of "grab" claw thorns. The claw thorn array has the function of automatically triggering the "trigger" and contracting and attaching when landing. The state before attachment is shown in Figure 7. It shows that when the restraint is released, the singlegroup claw mechanism automatically shrinks and grasps under the action of the compression spring, as shown in Figure 8. In actual working conditions, due to the complex topography of the asteroid surface, not all the 8 pairs of 


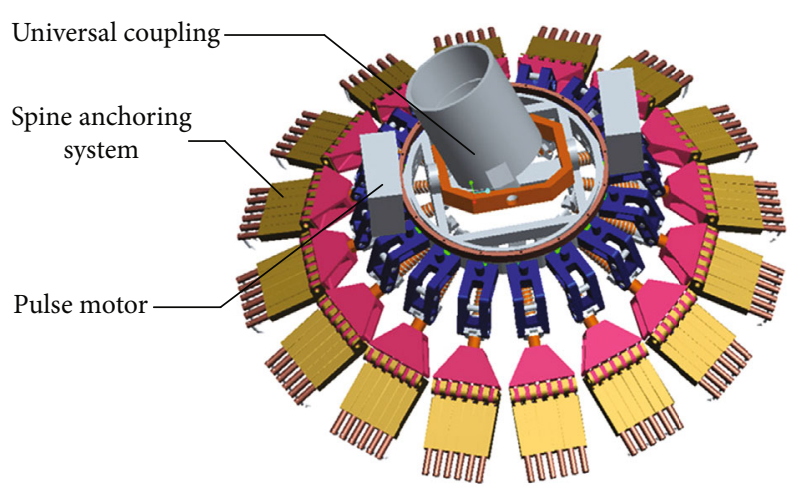

Figure 6: Composition of the spine anchoring system.

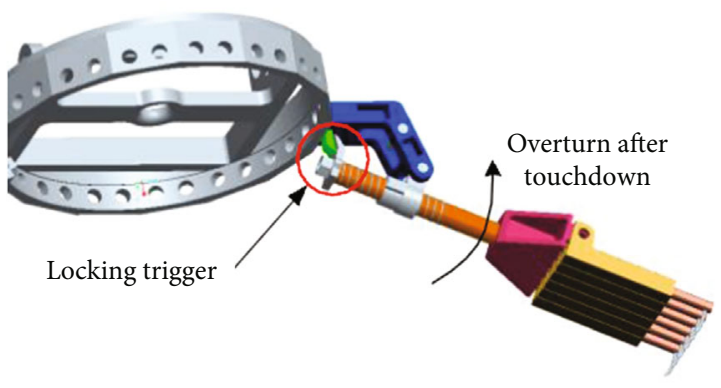

FIGURE 7: Initial installation status.

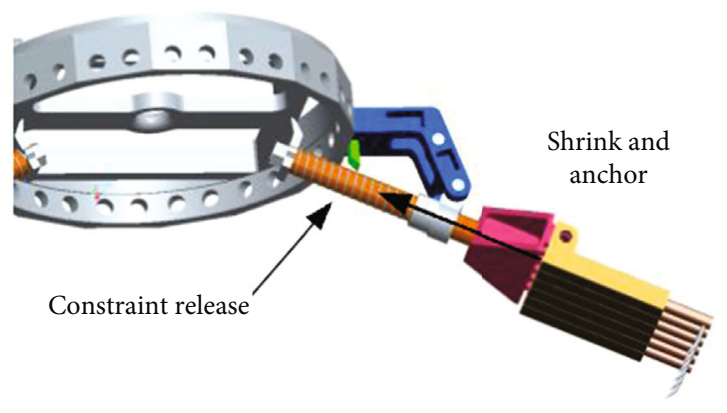

FIgUre 8: Poststatus.

"counter-grab" claws can be attached, and the 6 sets of thorn tips of each pair of "counter-grab" claws are not fully attached.

3.2. Working Principle of the Claw Attachment System. After the claw attachment system touches the surface of the asteroid, it automatically adjusts its position to adapt to the terrain of the attachment point through the universal joint mechanism. When the pressure transmitted by the landing buffer to the claw attachment system reaches a certain value, the claw attachment system automatically flips and shrinks to achieve adhesion on the hard and rough asteroid surface. As shown in Figure 9, the single-group claw thorn mechanism adopts a two-stage flexible connection design, the first level is the telescopic deformation of the thorn tip and the rotation within a certain angle range, and the second level is the telescopic deformation of the single claw-palm con-

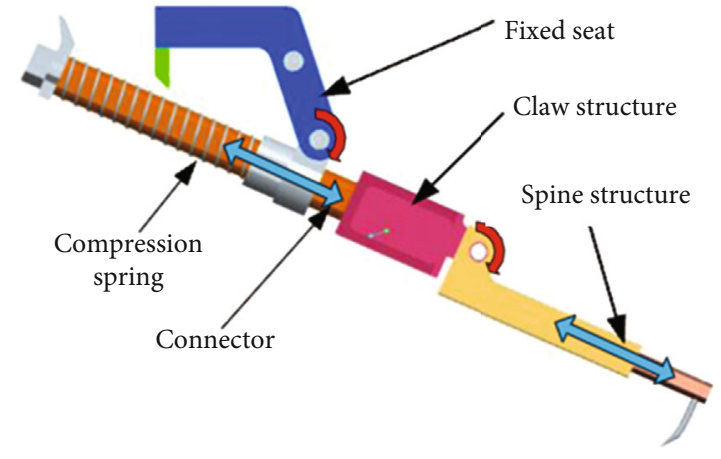

FIgURE 9: Mechanism of the spine anchoring system.

necting handle and the claw-palm structure and rotation within a certain angle range between the fixed seats.

During the landing process of the lander, the universal mechanism of the claw attachment mechanism will be pulled away from the surface of the asteroid. The claw attachment mechanism is flexibly connected with the inner cylinder of the landing buffer through its damping mechanism to buffer the impact force. After the landing attachment task is completed, the control system will drive the reset steering gear to rotate to realize the automatic reset of the claw attachment system after a delay of a period of time. It is used for the desorption or reattachment of the claw attachment system during the experiment. It can be applied to roaming detection on the surface of small celestial bodies.

\section{Analysis of the Biomimetic Adhesion Mechanism}

The force balance analysis of the claw-stab mechanism shown in Figure 10 can obtain the force balance equation of the claw-stab mechanism:

$$
F_{s} \cos \theta_{1}-F_{n} \sin \theta_{1}=k_{1} \Delta r_{1} \text {, }
$$

$$
F_{s} \sin \theta_{1} r_{1}+F_{n} \cos \theta_{1} r_{1}-M_{1}=0
$$

$$
F_{s} \cos \theta_{2}-F_{n} \sin \theta_{2}=k_{2} \Delta r_{2} \text {, }
$$

$$
F_{s} \sin \theta_{2} r_{2}+F_{n} \cos \theta_{2} r_{2}-M_{2}+M_{1}=0 \text {. }
$$

Among them, $F_{s}$ and $F_{n}$ are the tangential and normal contact forces between the claw-thorn mechanism and the attached surface.

$\theta_{1}, \theta_{2}$ are the angles between the claw thorn and the connecting handle and the horizontal direction.

$r_{1}$ and $r_{2}$ are the length of the claw and the connecting handle.

$k_{1} \Delta r_{1}, k_{2} \Delta r_{2}, M_{1}$, and $M_{2}$ are the restraining forces of springs and torsion springs. 


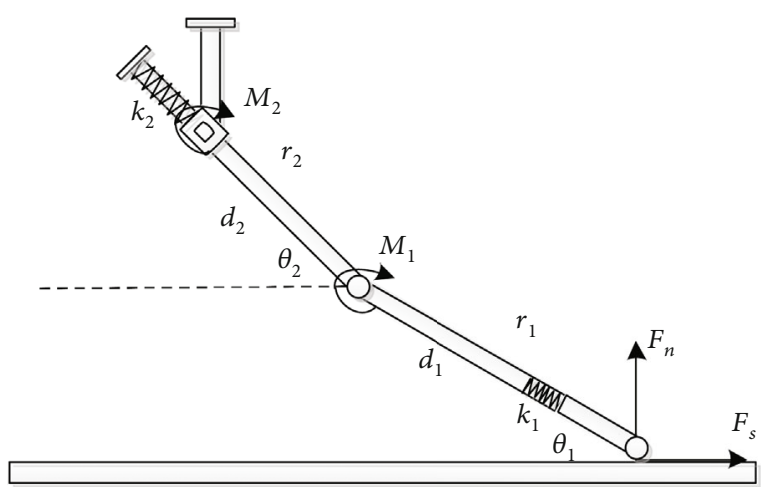

FIGURE 10: Force analysis of the spine anchoring system. lows:

The binding forces of spring and torsion spring are as fol-

$$
\begin{aligned}
& k_{1} \Delta r_{1}=k_{1}\left(r_{1}-r_{1}^{*}\right), \\
& k_{2} \Delta r_{2}=k_{2}\left(r_{2}-r_{2}^{*}\right), \\
& M_{1}=k_{\theta 1}\left(\theta_{1}-\theta_{1}^{*}\right), \\
& M_{2}=k_{\theta 2}\left(\theta_{2}-\theta_{2}^{*}\right) .
\end{aligned}
$$

From the first two equations in (1), the contact force between the claw-stab mechanism and the attached surface can be obtained as

$$
\left[\begin{array}{c}
F_{s} \\
F_{n}
\end{array}\right]=\left[\begin{array}{cc}
\cos \theta_{1} & \sin \theta_{1} \\
-\sin \theta_{1} & \cos \theta_{1}
\end{array}\right]\left[\begin{array}{c}
k_{1}\left(r_{1}-r_{1}^{*}\right) \\
\frac{1}{r_{1}} k_{\theta_{1}}\left(\theta_{1}-\theta_{1}^{*}\right)
\end{array}\right] .
$$

From the last two equations in (1),

$$
\begin{aligned}
{\left[\begin{array}{c}
F_{s} \\
F_{n}
\end{array}\right]=} & {\left[\begin{array}{cc}
\cos \theta_{2} & \sin \theta_{2} \\
-\sin \theta_{2} & \cos \theta_{2}
\end{array}\right] } \\
& \cdot\left[\begin{array}{c}
k_{2}\left(r_{2}-r_{2}^{*}\right) \\
\frac{1}{r_{2}}\left(k_{\theta 2}\left(\theta_{2}-\theta_{2}^{*}\right)-k_{\theta 1}\left(\theta_{1}-\theta_{1}^{*}\right)\right.
\end{array}\right] .
\end{aligned}
$$

Substituting (6) into (7) will get

$$
\begin{aligned}
& {\left[\begin{array}{cc}
\cos \theta_{1} & \sin \theta_{1} \\
-\sin \theta_{1} & \cos \theta_{1}
\end{array}\right]\left[\begin{array}{c}
k_{1}\left(r_{1}-r_{1}^{*}\right) \\
\frac{1}{r_{1}} k_{\theta_{1}}\left(\theta_{1}-\theta_{1}^{*}\right)
\end{array}\right]} \\
& \quad=\left[\begin{array}{cc}
\cos \theta_{2} & \sin \theta_{2} \\
-\sin \theta_{2} & \cos \theta_{2}
\end{array}\right]\left[\begin{array}{c}
k_{2}\left(r_{2}-r_{2}^{*}\right) \\
\frac{1}{r_{2}}\left(k_{\theta 2}\left(\theta_{2}-\theta_{2}^{*}\right)-k_{\theta 1}\left(\theta_{1}-\theta_{1}^{*}\right)\right.
\end{array}\right] .
\end{aligned}
$$

The coordinates of the connection point of the fixed seat are obtained from the geometric relationship of the claw
TABLE 1: Correlative parameter of the anchoring system.

\begin{tabular}{lcc}
\hline Parameter & Value & Remark \\
\hline$r_{1}{ }^{*}$ & $37.5 \mathrm{~mm}$ & \\
$r_{2}{ }^{*}$ & $36.5 \mathrm{~mm}$ & \\
$\theta_{1}{ }^{*}$ & $25^{\circ}$ & \\
$\theta_{2}{ }^{*}$ & $25^{\circ}$ & \\
$k_{1}$ & $0.15 \mathrm{~N} / \mathrm{mm}$ & Half of the spring works \\
$k_{2}$ & $0.32 \mathrm{~N} / \mathrm{mm}$ & \\
$k_{\theta 1}$ & $10 \mathrm{~N} / \mathrm{mm} / \mathrm{rad}$ & \\
$k_{\theta 2}$ & $668 \mathrm{~N} / \mathrm{mm} / \mathrm{rad}$ & \\
\hline
\end{tabular}

thorn mechanism:

$$
\begin{aligned}
& x=-r_{1} \cos \theta_{1}-r_{2} \cos \theta_{2}, \\
& y=r_{1} \sin \theta_{1}+r_{2} \sin \theta_{2} .
\end{aligned}
$$

Combine the 4 equations in (8) and (10). For this nonlinear equation system, you can use Newton-Raphson and other methods to solve to get $\theta 1, \theta 2, r_{1}$, and $r_{2}$ and then use (6) to calculate the claw mechanism adhesion.

The relevant parameters of the claw-stab mechanism are shown in Table 1. In the attachment process, it is assumed that only half (7) of the springs in the claws work. Use the method in the previous section to calculate the adhesion of the claw-stab mechanism. The displacement in both directions of the connecting position of the fixed seat is about $10 \mathrm{~mm}$. According to the above data, the normal adhesion force $F_{n}$ of the single claw mechanism on the contact surface is $4.68 \mathrm{~N}$.

\section{Experimental Verification of New Attachment Mechanism for Small Celestial Bodies}

In order to verify the gripping performance of the claws, an experimental verification study was carried out, focusing on the matching of the rigidity of the two-level flexible connection, and the adhesion and the probability of the gripping system of the claws were tested. As shown in Figure 11, the experiment uses volcanic rocks with a rough scale of centimeters (diameter greater than $40 \mathrm{~cm}$ ) to simulate the surface geological structure and topographic surface environment of hard asteroids and conduct the attachment process experiment of the claw attachment system. During the experiment, keeping the axis of the circular frame perpendicular to the surface of the volcanic rock, simulating the landing speed of the probe, pressing down the claw thorn system to the claw palm unit all actuate, and measuring the adhesion along the vertical direction. Through the groping experiment of the claw gripping mechanism, the spring stiffness at all levels is well matched. The dead weight of the claw gripping mechanism is $1.8 \mathrm{~kg}$, and the maximum tensile force measured by the spring balance experiment is $74.4 \mathrm{~N}$, and the gripping force can reach $56.4 \mathrm{~N}$ after removing its own weight. After 


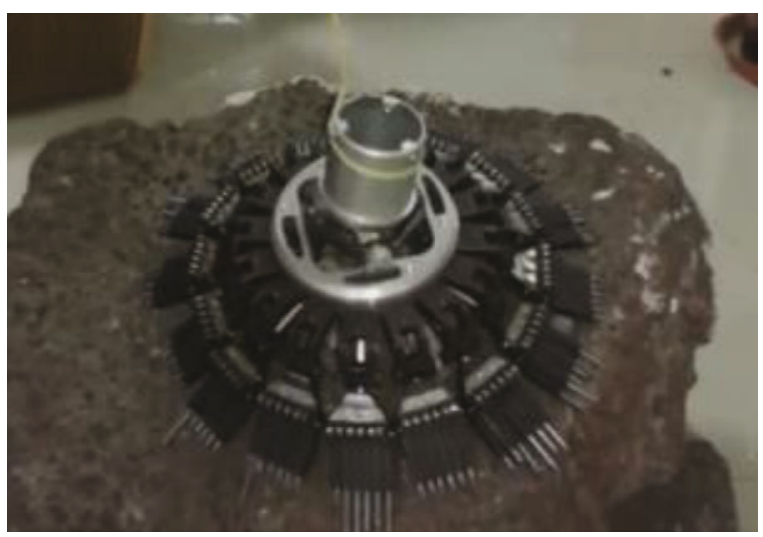

FIgURE 11: Anchoring experiment on the volcanic rock.

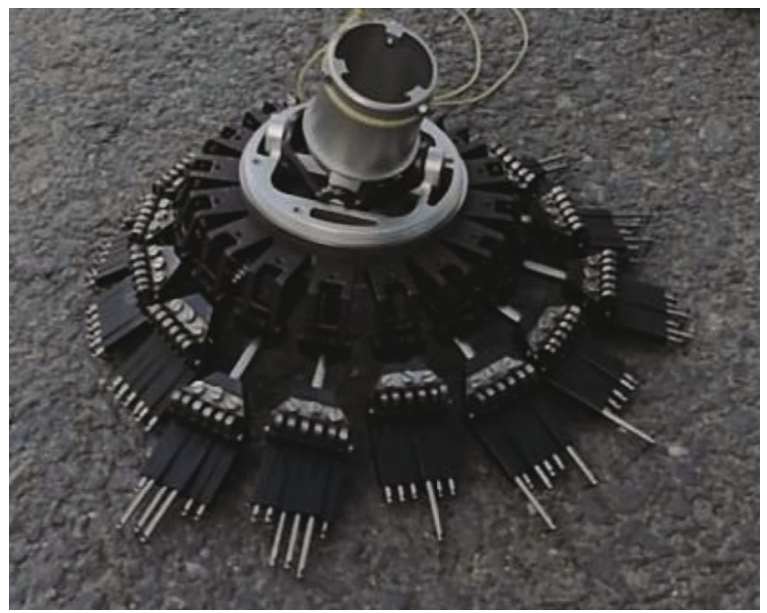

FIGURE 12: Anchoring experiment on the concrete slab surface.

analyzing the experimental claw piercing device, there are 13 groups that play a role of adhesion, the theoretical force value is $60.84 \mathrm{~N}$, and the analysis error is $7.9 \%$.

In order to verify the ultimate adhesion capacity of the claw stab device, as shown in Figure 12, the experiment selected the common asphalt pavement simulation medium to carry out the claw adhesion experiment. The experiment measured the maximum tensile force of $54.0 \mathrm{~N}$, and the gripping force can reach $36.0 \mathrm{~N}$ after the dead weight. After analyzing the experimental claw piercing device, there are 9 groups that play the role of adhesion, the theoretical force value is $42.12 \mathrm{~N}$, and the analysis error is $17.0 \%$.

\section{Summary}

This paper proposes a bionic beetle's claw-thorn attachment system, which can be applied to the relatively hard surface of asteroids for effective attachment and provide reliable adhesion. The research conclusions are as follows:

(1) A two-stage flexible connection claw spur component based on bionic claw spurs is proposed, with reasonable stiffness matching design and strong terrain matching adaptability
(2) For the force balance analysis of the claw-stab system, the contact force between the claw-stab mechanism and the attached surface is obtained based on the Newton-Raphson method, and the error between the analysis result and the experimental result is less than $17 \%$;

(3) Adhesion experiments were carried out to simulate the surface geological structure and terrain surface environment of hard asteroids. The adhesion force of volcanic rocks can reach $56.4 \mathrm{~N}$, and the adhesion force of ordinary asphalt roads can reach $36.0 \mathrm{~N}$

(4) The attachment system is based on the drive reset device, which can reset the attachment multiple times to meet the needs of multiple detection and roaming detection

\section{Data Availability}

The data used to support the findings of this study were supplied by the National Natural Science Foundation of China under license and so cannot be made freely available. Requests for access to these data should be made to Wang Yongbin, ybkindy@163.com.

\section{Conflicts of Interest}

The author(s) declare(s) that they have no conflicts of interest.

\section{References}

[1] P. Desai, "Entry dispersion analysis for the genesis sample return capsule," Journal of Spacecraft and Rockets, vol. 38, no. 3, pp. 345-350, 2001.

[2] J. P. Sanchez and C. R. Mclnnes, "Assessment on the feasibility of future shepherding of asteroid resources," Acta Astronautics, vol. 73, pp. 49-66, 2012.

[3] R. W. Farquhar, D. W. Dunham, and J. V. Mcadams, "NEAR mission overview and trajectory design," Journal of the Astronautical Sciences, vol. 43, no. 4, pp. 353-371, 1995.

[4] J. VEVERKA, B. FARQUHAR, M. ROBINSON et al., "The landing of the NEAR-shoemaker spacecraft on asteroid 433 Eros," Nature, vol. 413, no. 6854, pp. 390-393, 2001.

[5] R. NELSON, K. WHITTENBURG, and M. HOLDRIDGE, "433 Eros landing development of NEAR Shoemaker's controlled descent sequence," in 15th annual AIAA/USU conference on small satellites, pp. 1-11, Los Angeles, SSC, 2001.

[6] A. ACCOMAZZO and P. FERRI, "Rosetta visits asteroid (21) lutetia," in 61st International Astronautical Congress, p. 17, City of Light, CZ France, 2006.

[7] P. Ferri, "Mission operations for the new Rosetta," Acta Astronautica, vol. 58, no. 2, pp. 105-111, 2006.

[8] H. YANO, T. KUBOTA, H. MIYAMOTO et al., "Touchdown of the Hayabusa spacecraft at the Muses Sea on Itokawa," Science, vol. 312, no. 5778, pp. 1350-1353, 2006.

[9] Y. Tsuda, M. Yoshikawa, M. ABE, H. Minamino, and S. Nakazawa, "System design of the Hayabusa 2-asleroid sample return mission to 1999 ju 3," Acta Astronautica, vol. 9l, pp. 356-362, 2013. 
[10] A. FUJIWARA, T. MUKAI, J. KAWAGUCHI, and K. T. Uesugi, "Sample return Mission to NEA: MUSES-C," Advances in Space Research, vol. 25, no. 2, pp. 231-238, 2000.

[11] F. KAZUHISA, I. YOSHIFUMI, and H. KOIYU, Assessment of Dynamic Stability of Muses-C Capsule in Hypersonic Rarefied Regime: AIAA 2003-3893, AIAA, Washington D C, 2003.

[12] J. P. Bibring, "The rosetta lander ("philae") investigations," Space Science Reviews, vol. 128, no. 1-4, pp. 205-220, 2007.

[13] S. Ulamec, S. Espinasse, B. Feuerbacher et al., "Rosetta LanderPhilae: omplications of an alternative mission," Acta Astronautica, vol. 58, no. 8, pp. 435-441, 2006.

[14] P. Aaron and F. Matthew, Microgravity coring: a self-contained anchor and drill for consolidated rock, vol. 1335, IEEE AC, Shanghai, 2011.

[15] M. Murphy, "Waalbot II: adhesion recovery and improved performance of a climbing robot using fibrillar adhesives," The International Journal of Robotics Research, vol. 30, no. 1, pp. 118-133, 2011.

[16] T. A. Alan, "Designing compliant spine mechanisms for climbing," Journal of mechanisms and Robotics, vol. 4, 2012.

[17] S. A. Sipila, Z. C. Scoville, J. T. Bowie, and J. A. Buffington, "Extravehicular activity asteroid exploration and sample collection capability," in Int. Conf. on Space Oper, pp. 1-11, 2014.

[18] P. Aaron, "Demonstrations of gravity independent mobility and drilling on natural rock using microspines," in 2012 IEEE International Conference on Robotics and Automation, pp. 3547-3548, 2012. 